(C) 1979 IEEE. Personal use of this material is permitted. However, permission to reprint/republish this material for advertising or promotional purposes or for creating new collective works for resale or redistribution to servers

or lists, or to reuse any copyrighted component of this work in other works must be obtained from the IEEE.

IEEE Transactions on Nuclear Science, Vol. NS-26, No. 3, June 1979

\title{
ELECTRON MODEL EXPERIMENT TO STUDY INSTABILITIES IN LONG PERIODIC FOCUSING SYSTEMS FOR INTENSE BEAMS*
}

M. Reiser, W. Namkung, and M. A. Brennan†

\section{Abstract}

The proposed use of intense beams of high-energy heavy ions for pellet fusion have renewed interest in the problem of space-charge limits in long periodic focusing systems. Recent analytical and numerical studies at Berkeley and NRL for beams with a $\mathrm{K}-\mathrm{V}$ distribution indicate that instabilities may develop in such systems when the beam currents exceed certain thresholds. Since no relevant experimental data exists with which to compare the theory, we propose an inexpensive, small-scale experiment with a $5-20 \mathrm{kV}$ electron beam to study this problem. The electron beam is injected into a periodic solenoid focusing system consisting of 20 solenoid lenses initially. The pertinent results of beam transport theory are reviewed and the design features of the electron beam experiment are presented.

\section{Introduction and Motivation}

Recently, in connection with studics of heavy-ion accelerators for pellet fusion, instabilities in beam transport systems related to perturbations in the space-charge potential distribution have become a focus of interest. This particular type of instability had first been observed a decade ago by the late

$R$. Chasman ${ }^{1}$ in computer simulation studies of the proton beam in the initial stages of the Brookhaven 200-MeV linac. Chasman found that space-charge related effects caused a substantial growth of beam emittance (by factors of 2 to 3 ), most of which occurred in the initial sections of the quadrupole FODO lattice of the linac. Subsequent1y, R. Gluckstern ${ }^{2}$ developed an analytical theory of the oscillation modes in a round beam propagating through a long, uninterrupted, solenoidal focusing field.

The proposed use of heavy ion accelerators for pellet fusion led to renewed interest in the problem of high-current beam transport during the past two years. Maschke $^{3}$ derived a formula for the maximum beam current that can be transported in a FODO channel. An analytic theory was developed by Reiser, ${ }^{4}$ which clarified questions concerning this formula and the scaling laws 5 for heavy ion beam transport. In detailed studies, Laslett, Smith, and collaborators ${ }^{6}$ at the Lawrence Berkeley Laboratory have been examining the stability problem in FODO, interrupted solenoid, and long (continuous) solenoid channels, following the approach developed previously by Gluckstern. Paralle1ing this work are numerical simulation studies by Haber (in collaboration with Maschke) ${ }^{7}$ and also by Penner. ${ }^{8}$ Haber studied beam propagation through FODO channe1s (consisting of thin quadrupole lenses) using up to $1.6 \times 10^{4}$ macroparticles, and, like Chasman, he observed emittance growth by a factor 2 to 3 for various parameter regimes. Good agreement was found between his results and the results for unstable modes and growth rates obtained analytically by the Berkeley group. The numerical simulation revealed, furthermore, that the instabilities saturate after an initial growth.

A11 of this theoretical work so far is based on the $\mathrm{K}-\mathrm{V}$ distribution, the only known phase-space distribution that leads to linear force equations for the

\footnotetext{
†Electrical Engineering Department and Department of Physics and Astronomy, University of Maryland, College Park, Maryland 20742.
}

equilibrium beam. At present, no experimental data exists with which to compare the theory; thus, a systematic experimental investigation of the spacecharge related instabilities is called for.

The theoretically predicted growth rates for the instabilities depend on the beam intensity. As the current rises above the stability threshold, the growth rate rapidly reaches a maximum value, then decreases with increasing current. At or near the maximum, it typically takes only a few magnet periods of the focusing channel to obtain an e-folding growth in amplitude, whereas at higher beam intensities, it takes an increasingly longer distance for the instability to grow. Thus, for a systematic experimental study of the instabilities, one needs a sufficiently large number of magnet periods somewhere between 10 and 100 . For ion beams, such a channel becomes prohibitively long and expensive. It is for this reason that we propose to use electrons to study the physics of beam transport in periodic magnetic fields. ${ }^{9}$ In view of the compact size ( $3 \mathrm{~m}$ for 20 magnet periods) and low costs, an electronbeam model is a natural choice for such an experiment, and the results can be scaled to large magnet systems for any ion species from protons to uranium ions. Before describing the features of the proposed electron-beam facility, we will present in the next section a brief review of the theory of beam transport and instabilities.

\section{Brief Review of Beam Transport Theory}

The theory of periodic focusing was first developed by Courant and Snyder 10 and later extended by Kapchinskij and Vladimirskijll to include the effects of space charge. The latter introduced a phase-space distribution function, now generally referred to as the "K-V distribution," which has the property that its projections in any two-dimensional plane $\left(x-y, p_{x}-x\right.$, etc.) are ellipses with uniform particle density.

In the absence of space-charge forces, individual particles in a beam propagating throngh a periodic focusing system perform oscillations with wavelength $\lambda_{0}$. The phase shitt $\mu_{0}$ defines the fraction of a particle oscillation (for zero space charge) that occurs in one period $S$ of the lattice of periodically spaced lenses. According to the theory, stable motion exists only for $\left|\cos \mu_{0}\right| \leq 1$, i.e., $\mu_{0} \leq 180^{\circ}$. Space-charge effects result in a larger wavelength $\lambda>\lambda_{0}$, smaller phase shift $\mu<\mu_{0}$ per lattice period. The first studies of the Berkeley group showed that, due to the space charge, parametric instabilities of the beam envelope occur for $\mu_{0}>90^{\circ}$. These envelope instabilities (which had been observed already in much earlier work ${ }^{12}$ on periodic focusing of electron beams) then limit the operation of the system to $\mu_{0} \leq 90^{\circ}$. But even in the region below $90^{\circ}$, instabilities were found that are due to perturbations in the potential distribution of the beam. In the undisturbed $\mathrm{K}-\mathrm{V}$ beam, the potential is quadratic in $x$ and $y$. The Berkeley group examined perturbations in the space-charge potential, which involve terms of higher than quadratic order in $x$ and $y$ and obtained the following general results: 13,14 (1) Modes of 4 th and 6 th order perturbations lead to Instabilities in FODO channels, interrupted solenoid channels, and long solenoids, which can be avoided by operating in the range $\mu / \mu_{0} \geq 0.4$, the maximum current being given by 
$\mu_{0}=90^{\circ}$ and $\mu=36^{\circ}$. (2) Modes of third order cause instabllities in FODO and interrupted solenold channels at even lower beam intensities. (They are absent in long solenolds.) Specifically, for $\mu_{0}=90^{\circ}$, these third-order instabilities can be avolded only by operating the system at values $\mu \geq 57.5^{\circ}$. On the other hand, third-order instabllities do not occur when $\mu_{0} \leq 60^{\circ}$. (3) Below $\mu_{0}=60^{\circ}$, the intensity 11m1t is determined by the fourth and fifth order modes, 1.e., the region $\mu / \mu_{0} \geq 0.4$ is stable according to (1). The Berkeley group recommends that $\mu_{0}=60^{\circ}, \mu=24^{\circ}$ be chosen as operating parameters since the current in this case is larger than for $\mu_{0}=90^{\circ}, \mu=57.5^{\circ}$.

Let us now briefly discuss the implications of these results for the design of a focusing channel in the framework of the smooth approximation theory, 4 which is valid in the region $\mu_{0}<90^{\circ}$ for high-current beam transport. In solving the $K-V$ envelope equations for a matched beam, it is convenient to introduce in the space-charge term the "generalized perveance" $K=\left(I / I_{0}\right) 2 \beta^{-3} \gamma^{-3}$, where $I$ is the beam current and $I_{0}=4 \pi \varepsilon_{0} m_{0} c^{3} / q$ is the Alfvern-Lawson limiting current, 1.e., $3.1 \times 10^{7} \mathrm{~A} / \mathrm{Z}$ amperes for ions of charge number $\mathrm{Z}$ and mass number $A$, and $1.7 \times 10^{4}$ amperes for electrons. One then obtains for a matched beam the relation ${ }^{4}$

$$
K=\frac{I}{I_{0}} \frac{2}{B^{3} \gamma^{3}}=\frac{\mu_{0}}{S} \alpha\left[1-(\varepsilon / \alpha)^{2}\right],
$$

where $\alpha \pi$ is the acceptance of the channel for zero space charge and $\varepsilon \pi$ is the emittance. If $\ell$ denotes the length of a nagnet, $L$ the free space between magnets, the period of a FODO channel is $S=2 \ell(1+\mathrm{L} / \ell)$, and the strength of the magnets is measured by the focusing parameter $\theta=\left(\mathrm{qB}_{0} / \mathrm{m}_{\mathrm{o}} \mathrm{c} \beta \gamma \mathrm{\gamma}\right)^{1 / 2} \ell$. The phase shift $\mu_{0}$ is a function of $\theta$ and $L / l$. The acceptance parameter $\alpha$, on the other hand, is defined as $\alpha=a^{2} / w_{0}^{2}, \max$, where $w_{0}, \max$ denotes the maximum of the characteristic amplitude function $w_{0}(s)$ for the periodic channel in the case of zero space charge. Like $\mu_{O}, w_{O}$, max is also a function of $\theta$ and $L / l$ that can be calculated analytically. The phase shift $\mu$ of the particle oscillations and the amplitude function w(s) with space charge can be calculated in the smooth-approximation theory by introducing the dimensionless space-charge parameter $u=K S / 2 \mu_{0} \varepsilon$. One obtains the expressions

$$
w(s)=w_{0}(s)\left[u+\sqrt{1+u^{2}}\right]^{1 / 2}
$$

and

$$
\mu=\mu_{0}\left[\sqrt{1+u^{2}}-u\right] .
$$

Furthermore, one finds that $\varepsilon / \alpha=\mu / \mu_{0}$, or, in view of (3),

$$
\alpha=\frac{\varepsilon}{\mu / \mu_{0}}=c\left[\sqrt{1+u^{2}}-u\right]^{-1} \text {. }
$$

If $\mu_{0}$ is given and $\mu / \mu_{0}$ is fixed due to Instabilities, then all the other parameters are uniquely determined for any given choice of the lattice ratio $\mathrm{L} / \mathrm{l}$. One then obtains for the beam current with lons of charge number $Z$ and mass number $A$ the relation (MKS unfts)

$$
I_{[A]}=3.64 \times 10^{6} \mathrm{C}\left(\frac{Z}{A}\right)^{1 / 3}(B \gamma)^{7 / 3} \mathrm{~B}_{\mathrm{O}}^{2 / 3} \varepsilon^{2 / 3}
$$

which is in the form first suggested by Maschke. ${ }^{3}$ The constant C is identical with the "figure of merit" (FM) of the Berkeley group. From the smooth-approximation theory, one obtains the result

$$
C=F M=\frac{\mu_{0}}{1+L / l} \frac{1-\left(\mu / \mu_{0}\right)^{2}}{\left(\mu / \mu_{0}\right)^{2 / 3}} \frac{1}{\theta^{4 / 3}\left(\frac{w_{0}, \max }{\sqrt{l}}\right)^{2 / 3}} .
$$

For a given cholce of $\mu_{0}$ and $L / l$, the parameters $\theta$ and $\mathrm{w}_{0, \max } / \sqrt{\ell}$ are fixed and the beam current in a FODO channel is seen to vary as

$$
\text { I } \propto\left[1-\left(\mu / \mu_{0}\right)^{2}\right] /\left(\mu / \mu_{0}\right)^{2 / 3} .
$$

In the case of interrupted solenoids, we have $S=\ell(1+L / l)$ and $\theta=q B_{S} l / 2 m_{0} c B \gamma$ where $B_{S}$ is the solenoldal fleld strength. In place of $(6)$, one obtains then for the lon current limit the relation

$$
\begin{aligned}
& I_{[A]}=2.48 \times 10^{6} C_{S} \beta^{2} \gamma^{2} B_{S} \varepsilon, \\
& C_{S}=\frac{\mu_{0}}{(1+L / \ell) \theta} \frac{1-\left(\mu / \mu_{0}\right)^{2}}{\mu / \mu_{0}} .
\end{aligned}
$$

For electrons, the numerical factor in (8) is different and we have

$$
I_{[A]}=1.36 \times 10^{3} C_{S} \beta^{2} \gamma^{2} B_{S} \varepsilon .
$$

\section{Description of Proposed Facility}

We propose to study the physics of beam transport in long periodic focusing systems for conditions where the space charge of the beam has a significant effect on the particle motion. By long systems, we mean a sufficiently large number of focusing lenses that instabilities of the type discussed above can be observed experimentally. A number of 20 periods appears to be a good initial goal, with more lenses to be added later if desired. By signiftcant spacecharge effects, we mean that the self fields of the beam increase the wavelength of the particles' oscillations by a factor 2 or more, i.e., $\mu / \mu_{0}<0.5$ or $u>0.75$. In addition, one wants to be able to change parameters over as wide a range as possible to study, for instance, how the instabilities and the associated growth rates vary with the space-charge intensity. The proposed facility will satisfy these requirements. Electron beams generally have a very small emittance (or transverse temperature), i.e., u is significantly larger than 0.75 . However, we plan to decrease u by passing the beam through a thin wire mesh, which reduces the beam current and increases the emittance due to scattering.

A major advantage of the proposed facility is the long pulse length of the beam (design goal is between 1 and $10 \mu s$ ) and the absence of accelerating structures and external electric fields. In linear accelerators, the rapid acceleration of the particles and coupling effects between longitudinal and transverse motion makes an analysis of the problem very difficult, as $R$. Chasman pointed out already. ${ }^{1}$ The electron beam in the proposed experiment, on the other hand, is essentially two-dimensional so that a direct comparison between experiment and theory should not be too difficult.

Figure 1 shows a schematic layout of the proposed experimental facility. The electron gun is a scaleddown version (ratio $1: 5$ ) of the $60-\mathrm{kV}$ klystron gun designed by $W$. Herrmannsfeldt at Stanford (SLAC) for the PEP project. It has a perveance of $\mathrm{k}=\mathrm{I} / \mathrm{V}^{3} / 2=$ $0.75 \times 10^{-6}$ (amps/volt ${ }^{3 / 2}$ ), 1.e., the current is $0.75 \mathrm{~A}$ at $10 \mathrm{kV}$. To reduce power consumption, a pulsed high-voltage supply will be used with a tentative pulse length of a few microseconds and a repetition rate of $60 \mathrm{~Hz}$. The cathode radius is $1.3 \mathrm{~cm}$ and the focus occurs at approximate $1 \mathrm{y} 6.4 \mathrm{~cm}$ from the cathode where the beam radius is $0.57 \mathrm{~cm}$. The beam then passes through an iron plate, which shields the cathode from the solenoidal magnetic field. On the upstream side of the iron plate is the emittance/current 
control mesh discussed above. On the downstrean side is a spectal coil (or coil system) to be designed for matching the beam envelope. Following that is the periodic lattice of solenoids. We have already built three test solenoids with iron shields to obtain a well-defined, pronounced field variation. These coils are show in Fig. 1. The measured magnetic field variation on the axis is plotted in Fig. 2(a); the length of one magnet period in this case is $15 \mathrm{~cm}$. However, we plan to build the system such that the spacing between magnets can be varied easily. The preliminary design parameters for the facility are summarlzed in Table 1. The diagnostic elements consisting of pinhole plates, Faraday cup, and fluorescent screen will be mounted inside a diagnostics chamber from where they can be moved along or perpendicular to the beam direction. At the entrance of the diagnostics chamber is a special coil to be used for focusing the beam on the screen. Our plans are to assemble the solenoidal focusing coils in steps. Starting with a few colls, the beam profile will be carefully investigated, then more coils will be added and the measurements will be repeated at the larger distance downstream. The onset of an instability should show up in the shape of the beam cross-section profile on the fluorescent screen. When such an instability is observed, a more detailed study of the beam emittance will be made.

The main objective of this experiment is to identify all effects that contribute to a significant growth of the emittance such as beam mismatch, aberrations due to nonlinear magnetic field components, alignment errors, etc. Of particular importance is the region near the gun. A major reason for choosing the PEP gun design is the fact that it produces a lowconvergence, high-quality beam. This will, hopefully, avoid the "translaminar" flow problems that Brewer found in his study of beam propagation through a solenoid with uniform field. ${ }^{15}$ Special attention must also be given to the possibility that microwaves are generated along the beam, which could lead to deterioration of beam quality.

As an aid for the design of the facility and for proper matching of the beam, we have developed a computer program that integrates the $K-V$ envelope equations. In our solenoid case, the envelope radius $R$ versus distance $z$ obeys the equation

$$
\frac{d^{2} R}{d z^{2}}+k_{S}(z) R-\frac{K}{R}-\frac{\varepsilon^{2}}{R^{3}}=0,
$$

where $k_{S}(z)=\left[q B_{S}(z) / 2 m_{0} c \beta \gamma\right]^{2}$, and $B_{S}(z)$ is the magnetic field on the axis. Setting $\varepsilon=0$ and $k=0$, the program first calculates a single-particle trajectory to determine the phase shift $\mu_{0}$. With proper values of $\varepsilon$ and $K$, the matched envelope radius $R(z)$ is then calculated and plotted. Fig. 2(b) shows a plot of $R(z)$ from a computer run for the experimental field shape $B_{S}(z)$ in Fig. $2(a)$. The parameter values for this case were $\mu_{0}=90^{\circ}, \mu \approx 30^{\circ}$, peak field $B_{O}=142 \mathrm{G}$, lens period $\mathrm{S}=15 \mathrm{~cm}$, electron energy $\mathrm{T}=10 \mathrm{keV}$.

\section{References}

1. R. Chasman, IEEE Trans. NS-16, 202 (1969).

2. R. L. Gluckstern, Proc. 1970 Proton Linac Conf., Batavia, I11., p, $811 \mathrm{ff}$.

3. ERDA Summer Study of Heavy Ions for Inertial Fusion, LBL-5543, Dec. 1976, p. 72.

4. M. Reiser, Particle Accel. 8, 167 (1978).

5. Proc. Heavy Ion Fusion Workshop, BNL 50769, 0ct. 1977 , p. $115 \mathrm{ff}$.

6. L. Smith, HIFAN-13, S. Chat topadhyay, HIFAN-14, I. Hofmann and L. Jackson Laslett, HIFAN-14, Lawrence Berkeley Laboratory, Oct. 1977.
7. I. Haber and A. W. Maschke, NRL Memo. Repts. 3787 , May 1978 , and 3817 , July 1978.

8. S. Penner, in Ref. 5, p. 127.

9. M. Relser, W. Namkung, M. A. Brennan, W. Herrmannsfeldt, I. Hofmann, Bul1. APS 23, 770 (1978).

10. E. D. Courant and H. S. Snyder, Ann. Phys. 3 , 1 (1978)

11. I. M. Kapchinskij and V. V. Vladimirskij, Proc. Int. Conf. High Energy Acce1., CERN, Geneva, 1959, p. 274.

12. See, for example, J. R. Pierce, "Theory and Design of Electron Beams" (D. Van Nostrand Co., N.Y., 1954).

13. L. Jackson Laslett, in Ref. 5, p. $112 \mathrm{ff}$.

14. J. Bisognano, L. J. Laslett, L. Smith, USID-8079, HIFAN-43, Sept. 1978.

15. G. R. Brewer, J. App1. Phys. 30, 1022 (1959).

TABLE 1

\section{Preliminary Design Parameters for EBTE}

Phase shift per period without space charge $\mu_{0}$ $60^{\circ}-90^{\circ}$

Beam current, I $0.1-2.0 \mathrm{~A}$

Electron Energy, T $5-20 \mathrm{keV}$

Mean Beam Radius, $\mathrm{R}_{\mathrm{b}}$

$0.63-0.95 \mathrm{~cm}$

Axial length of test magnet, $\ell_{\exp }$

$6.8 \mathrm{~cm}$

Magnet 1attice period, $S$ $10-20 \mathrm{~cm}$ (adjustable)

Maximum peak $B_{z}$ field, $B_{0}$ 100-300 G (adjustable)

Total number of magnets

20 initially

*Work supported by the U.S. Department of Energy.

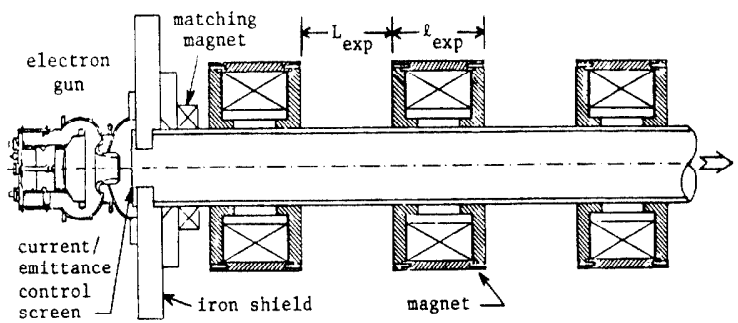

Fig. I Schematic of electron beam transport experiment.
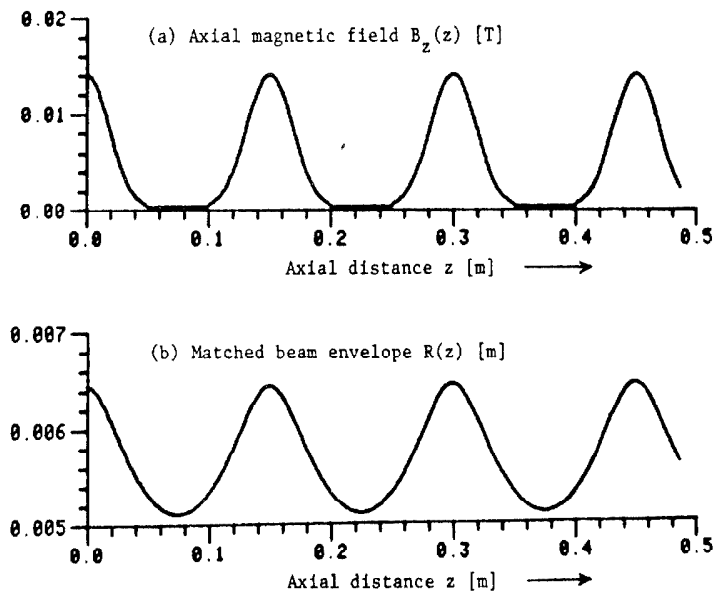

Fig. 2 The axial magnetic field along the axis for $H_{0}=90^{\circ}$ (a) and the matched beam envelope radius for $\mu / \mu_{0}=0.3$ (b). 